\title{
蓝光材料 $\operatorname{Ir}(I I I)$ 配合物的磷光效率与光谱的振动关联函数研究
}

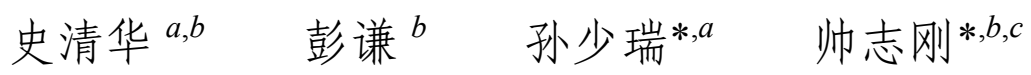 \\ ( ${ }^{a}$ 北京工业大学环境与能源工程学院 北京 100124) \\ $\left({ }^{b}\right.$ 中国科学院化学研究所 北京分子科学国家实验室 有机固体院重点实验室 北京 100190) \\ (c清华大学化学系 有机光电子与分子工程重点实验室 北京 100084)
}

\begin{abstract}
摘要 由于发光效率低和稳定性差, 蓝色磷光材料一直是发光材料研究领域的瓶颈. 为了更深层次地理解蓝色磷光分 子结构与发光效率之间的关系, 本工作结合密度泛函理论, 运用作者新近发展的系间窝越速率的振动关联函数计算方 法, 定量研究了新型蓝光发射分子 fac-tris(2-(4,6-difluorophenyl)pyridyl iridium $\left(f a c-\operatorname{Ir}\left(\mathrm{F}_{2} \mathrm{ppy}\right)_{3}\right)$ 的磷光光谱、辐射跃迁和 无辐射跃迁速率及其与温度的依赖关系, 计算结果很好地解释了实验测量结果. 计算表明: (1)相较于未取代的绿光材 料 $f a c-$ Ir (ppy $)_{3}$, 杂原子 $\mathrm{F}$ 的引入增加了 $\mathrm{T}_{1}$ 与 $\mathrm{S}_{0}$ 的能隙, 使得光谱蓝移, 但没有带来额外的分子结构弛豫的重整能, 从 而使得该蓝色材料保持了高的发光效率; (2)无辐射跃迁过程所耗散的电子激发态能量主要是通过配体 L1 中的连接氟 化苯环和吡啶环的 $C(5)-C(46)$ 键、吡啶环内 $C(43)-C(44)$ 键和 $C(42)-C(47)$ 键及氟化苯环内的 $C(3)-C(6)$ 键的伸缩振 动，因此，理论研究表明可以通过分子设计来抑制这些振动来进一步提高这类材料的发光效率.
\end{abstract}

关键词 系间窝越速率理论; 铱(III)配合物分子设计; 磷光光谱; 磷光量子效率

\section{Vibration Correlation Function Investigation on the Phosphorescence Quan- tum Efficiency and Spectrum for Blue Phosphorescent Ir(III) Complex}

\author{
Shi, Qinghua ${ }^{a, b}$ \\ Peng, Qian ${ }^{b}$ \\ Sun, Shaorui*,a \\ Shuai, Zhigang *,b,c
}

$\left({ }^{a}\right.$ College of Environmental and Energy Engineering, Beijing University of Technology, Beijing 100124)

$\left({ }^{b}\right.$ Key Laboratory of Organic Solids, Beijing National Laboratory for Molecular Science, Institute of Chemistry, Chinese Academy of Sciences, Beijing 100190)

( ${ }^{c}$ Key Laboratory of Organic Optoelectronics and Molecular Engineering, Department of Chemistry, Tsinghua University, Beijing 100084)

\begin{abstract}
A challenging issue for white organic light-emitting diodes is to improve the luminescence efficiency and stability for blue phosphorescence materials. In order to deeper understand the relationship between molecular structure and luminescence quantum efficiency, we apply our recently developed correlation function method coupled with density functional theory calculation to investigate the photophysical properties of $f a c$-tris(2-(4,6-difluorophenyl)pyridyl iridium $\left(f a c\right.$ - $\left.\operatorname{Ir}\left(\mathrm{F}_{2} \text { ppy }\right)_{3}\right)$, including phosphorescence emission spectra, radiative and nonradiative decay rates, and excited-state decay lifetime at different temperatures. All the calculated results can well reproduce the available experimental measurements. We further analyze the relevance of molecular parameters governing the photophysical processes. We found out that in $f a c-\operatorname{Ir}\left(\mathrm{F}_{2} \mathrm{ppy}\right)_{3}:(1)$ when compared with the archetypal green $f a c-\operatorname{Ir}(\mathrm{ppy})_{3}$, the introduction of $\mathrm{F}$ atoms can enlarge the energy gap between the excited triplet $T_{1}$ and the ground state $S_{0}$ resulting in blue-shift. This does not introduce extra reorganization energies in the excited-state relaxation process. So the blue phosphorescent $f a c-\operatorname{Ir}\left(\mathrm{F}_{2} \mathrm{ppy}\right)_{3}$ can exhibit high luminescence efficiency; (2) the main channels for nonradiatively dissipating the electronic excited-state energy are found to be the stretching vibrations of carbon and carbon bonds, such as $\mathrm{C}(5)-\mathrm{C}(46)$ linking the fluorophenyl and pyridyl rings, $\mathrm{C}(43)-\mathrm{C}(44)$ and $\mathrm{C}(42)-\mathrm{C}(47)$ of pyridyl ring, and $\mathrm{C}(3)-\mathrm{C}(6)$ of fluorophenyl ring in L1 ligand. This points out the direction for further increasing the light-emitting efficiency through suppressing these motions.
\end{abstract}

Keywords theory of intersystem crossing; molecular design of iridium (III) complex; phosphorescence spectrum; phosphorescence quantum efficiency

\section{1 引言}

近年来，由于有机电致发光二极管(OLED)在固态
照明和平板显示上展现出巨大的使用价值, 吸引着人们 对新型高效的电致发光材料的开发和研究. 电致磷光材 料因具有良好的发光性能和理论上可以达到 $100 \%$ 的内

*E-mail: sunsr@bjut.edu.cn, zgshuai@tsinghua.edu.cn

Received January 22, 2013; published March 26, 2013.

Project supported by the National Natural Science Foundation of China (Nos. 21290190, 91233105) and the Ministry of Science and Technology of China through 973 Program (Nos. 2009CB623600, 2013CB834703).

项目受国家自然科学基金(Nos. 21290190, 91233105)和科技部 973 项目(Nos. 2009CB623600, 2013CB834703)资助. 
量子效率深受到人们的无比青睐 ${ }^{[1,2]}$. 为此, 人们开发合 成了大量含重金属如铂 $(\mathrm{Pt})$ 、铱 $(\mathrm{Ir})$ 、锇 $(\mathrm{Os})$ 、铕 $(\mathrm{Eu})$ 的 有机配合物磷光材料, 颜色覆盖了整个可见光区 ${ }^{[3 \sim 5]}$, 其中铱(III)配合物最具有竞争力 ${ }^{[6 \sim 8]}$. 但是, 实验发现: 在蓝、绿和红三基色材料中, 绿光磷光材料性能已经成 熟稳定, 而红光和蓝光磷光材料都存在有效率低、稳定 性差等问题. 也就是说, 相对于绿光磷光材料, 波长红 移和蓝移都伴随着量子发光效率的降低 ${ }^{[9,10]}$. 这势必极 大程度地制约着 OLED 的快速发展. 所以, 如果能够深 层次理解其发光机理, 剖析分子微观结构对辐射跃迁和 无辐射跃迁速率的影响, 建立详尽的构效关系等显得非 常重要.

不同于传统的荧光材料分子的单线态发光, 磷光材 料分子是三线态发光, 即通过自旋轨道耦合 (spin-orbit coupling, SOC)作用来诱导三线态衰减到单线基态. 这 里有两个过程: 辐射过程和无辐射衰减过程, 两者的竞 争决定了磷光效率. 辐射衰减速率可以通过简单的爱因 斯坦自发辐射关系式结合量化计算得到 ${ }^{[11]}$. 无辐射跃 迁过程中, 除了能隙因素, 核构型的变化程度对其速率 大小的影响也是不可忽视的. 常用的有机过渡金属配合 物的磷光分子, 一般包括 $>40$ 个原子, 其激发三线态的 衰减时间尺度是微秒或毫秒级. 目前的电子动力学理论 还只是局限于小分子体系, 并且在时间尺度上只能到 $10 \mathrm{ps}$, 远远达不到发光过程的发生. 因此, 对于白光照 明分子这么大的体系, 要进行这么长时间的非绝热动力 学模拟是不可能的. 众多的振动模式和不同模式之间的 混合使得利用传统方法进行 Franck-Condon 积分计算的 计算量也远远超过了现有的计算能力, 所以即便是简单 的线性位移谐振子模型也无法对其进行定量描述.

最近，彭谦等 ${ }^{[12]}$ 发展了将自旋-轨道耦合与非绝热 耦合相结合的微扰理论, 为计算系间窝越速率和磷光速 率和光谱提供了普适性的公式. 该理论形式由于采用了 振动关联函数, 借助高维积分的解析推导和快速傅里叶 变换, 使得计算量随分子尺度从指数增长极大地降到仅 仅是矩阵运算的 $\mathrm{N}^{3}{ }^{[13]}$, 并首次实现了复杂的有机过渡 金属配合物的磷光效率的定量预测 ${ }^{[12]}$. 磷光材料中, fac-tris(2-(4,6-difluorophenyl)pyridyl iridium (fac$\left.\operatorname{Ir}\left(\mathrm{F}_{2} \mathrm{ppy}\right)_{3}\right)$ 是一个常见的蓝光磷光材料, 已经得到详尽 的实验研究和简单的理论计算 ${ }^{[7,14 \sim 16]}$. 因此, 我们以此 为例, 定量预测其光物理性质, 包括不同温度下的磷光 光谱、辐射和无辐射衰减速率、磷光效率及激发态寿命 等. 进一步微观分析一些重要参数, 可找出无辐射耗散 激发态能量的主要通道, 为实验上的分子改造和设计出 高效蓝色磷光材料提供依据和思路.

\section{2 理论方法}

\section{1 系间窝越的速率公式}

在二级微扰理论和玻恩-奥本海默(BO)绝热近似下,
无辐射跃迁速率可以表示为 ${ }^{[17]}$

$k_{\mathrm{f} \leftarrow \mathrm{i}}=\frac{2 \pi}{\hbar} \sum_{\nu, \mu} P_{\mathrm{i} \nu}\left|H_{\mathrm{f} \mu, \mathrm{i} \nu}^{\prime}+\sum_{n, \kappa} \frac{H_{\mathrm{f} \mu, n \kappa}^{\prime} H_{n \kappa, \mathrm{i} v}^{\prime}}{E_{\mathrm{i} \nu}-E_{n \kappa}}\right|^{2} \delta\left(E_{\mathrm{i} \nu}-E_{\mathrm{f} \mu}\right)$

其中, $\mathrm{i}, \mathrm{f}$ 分别表示电子的初态和末态, 对应的振动分别 是 $v$ 和 $\mu ; P_{\mathrm{i} v}$ 为初态振动态的波尔兹曼分布函数; $H^{\prime}$ 包含 非绝热耦合项和自旋轨道耦合项:

$\hat{H}^{\prime} \Psi_{\mathrm{i} v}=\hat{H}^{\mathrm{BO}} \Phi_{\mathrm{i}}(\mathbf{r} ; \mathbf{Q}) \Theta_{\mathrm{i} v}(\mathbf{Q})+\hat{H}^{\mathrm{SO}} \Phi_{\mathrm{i}}(\mathbf{r} ; \mathbf{Q}) \Theta_{\mathrm{i} v}(\mathbf{Q})$

$\hat{H}^{\mathrm{BO}}$ 为波恩-奥本海默非绝热耦合项，来自于 $\mathrm{BO}$ 近似 所忽略的原子核的动能, $\Phi$ 是电子态波函数, $\Theta$ 是原子核 波函数. 在一般的情况下, 忽略小量项, 耦合矩阵元可 以表示为:

$\left\langle\Phi_{\mathrm{f}} \Theta_{\mathrm{f} \mu}\left|\hat{H}^{\mathrm{BO}}\right| \Phi_{\mathrm{i}} \Theta_{\mathrm{i} v}\right\rangle=-\hbar^{2} \sum_{k}\left\langle\Phi_{\mathrm{f}} \Theta_{\mathrm{f} \mu} \mid \frac{\partial \Phi_{\mathrm{i}}}{\partial Q_{\mathrm{f} k}} \frac{\partial \Theta_{\mathrm{i} v}}{\partial Q_{\mathrm{f} k}}\right\rangle=$
$\sum_{k}\left\langle\Phi_{\mathrm{f}} \Theta_{\mathrm{f} \mu} \mid\left(\hat{P}_{\mathrm{f} k} \Phi_{\mathrm{i}}\right)\left(\hat{P}_{\mathrm{f} k} \Theta_{\mathrm{iv}}\right)\right\rangle$

$\hat{H}^{\mathrm{SO}}$ 为自旋轨道耦合项; $\mathbf{r}$ 和 $\mathbf{Q}$ 分别表示电子坐标和核 正则坐标; $\hat{P}_{\mathrm{f} k}$ 是末态第 $k$ 个振动模式的质量权重的动 量算符.

将式(2)和(3)代入(1), 利用 “康登” 近似可以得到:

$$
\begin{aligned}
& k_{\mathrm{f} \leftarrow \mathrm{i}}=k_{\mathrm{f} \leftarrow \mathrm{i}}^{(0)}+k_{\mathrm{f} \leftarrow \mathrm{i}}^{(1)}+k_{\mathrm{f} \leftarrow \mathrm{i}}^{(2)}, \\
& k_{\mathrm{f} \leftarrow \mathrm{i}}^{(0)}=\frac{2 \pi}{\hbar} R_{\mathrm{fi}}^{\mathrm{isc}} \sum_{v, \mu} P_{\mathrm{i} v}\left|\left\langle\Theta_{\mathrm{f} \mu} \mid \Theta_{\mathrm{i} v}\right\rangle\right|^{2} \delta\left(E_{\mathrm{i} v}-E_{\mathrm{f} \mu}\right) \\
& k_{\mathrm{f} \leftarrow \mathrm{i}}^{(1)}=2 \operatorname{Re}\left[\sum_{k} \frac{2 \pi}{\hbar} R_{\mathrm{fi}, k}^{\mathrm{isc}} \sum_{v, \mu} P_{\mathrm{i} v}\left\langle\Theta_{\mathrm{f} \mu}\left|\Theta_{\mathrm{i} v}\right| \hat{P}_{\mathrm{f} k} \mid \Theta_{\mathrm{f} \mu}\right\rangle\left(E_{\mathrm{i} v}-E_{\mathrm{f} \mu}\right)\right] \\
& k_{\mathrm{f} \leftarrow \mathrm{i}}^{(2)}=\sum_{k, l} \frac{2 \pi}{\hbar} R_{\mathrm{fi}, k l}^{\mathrm{isc}} \sum_{v, \mu} P_{\mathrm{i} v}\left\langle\Theta_{\mathrm{f} \mu}\left|\hat{P}_{\mathrm{f} l}\right| \Theta_{\mathrm{f} k} \mid \Theta_{\mathrm{i} v}\right\rangle \delta\left(E_{\mathrm{i} v}-E_{\mathrm{f} \mu}\right)
\end{aligned}
$$

这里

$$
R_{\mathrm{fi}}^{\mathrm{isc}} \equiv\left|H_{\mathrm{fi}}^{\mathrm{so}}\right|^{2} \equiv\left|\left\langle\Phi_{\mathrm{f}}\left|\hat{H}^{\mathrm{so}}\right| \Phi_{\mathrm{i}}\right\rangle\right|^{2}
$$

$R_{\mathrm{fi}, k}^{\mathrm{isc}} \equiv H_{\mathrm{fi}}^{\mathrm{SO}} T_{\mathrm{if}, k}$

$R_{\mathrm{fi}, k l}^{\mathrm{isc}} \equiv T_{\mathrm{if}, k} T_{\mathrm{fi}, l}$

$$
T_{\mathrm{if}, k} \equiv \sum_{n}\left(H_{\mathrm{i} n}^{\mathrm{SO}} \frac{\left\langle\Phi_{n}\left|\hat{P}_{\mathrm{f} k}\right| \Phi_{\mathrm{f}}\right\rangle}{E_{\mathrm{i}}-E_{n}}+\frac{\left\langle\Phi_{\mathrm{i}}\left|\hat{P}_{\mathrm{f} k}\right| \Phi_{n}\right\rangle}{E_{\mathrm{i}}-E_{n}} H_{n \mathrm{f}}^{\mathrm{SO}}\right)
$$

$$
\beta=1 /\left(k_{\mathrm{B}} T\right)
$$

又因为波尔兹曼分布函数, 


$$
P_{\mathrm{i} \nu}=\prod_{k} e^{-\beta E_{v_{k}}^{\mathrm{i}}} / Z_{\mathrm{i}}=e^{-\beta E_{v}^{\mathrm{i}}} / Z_{\mathrm{i}}
$$

其中, $Z_{\mathrm{i}}^{-1}=\sum_{v=\left\{0_{1}, 0_{2}, \cdots, 0_{N}\right\}}^{\infty} \mathrm{e}^{-\beta E_{v}^{i}}$ 是配分函数.

及 $\delta$ 函数的傅里叶变换:

$$
\delta\left(E_{\mathrm{if}}+E_{v}^{\mathrm{i}}-E_{\mu}^{\mathrm{f}}\right)=\frac{1}{2 \pi} \int_{-\infty}^{\infty} \mathrm{d} \tau \mathrm{e}^{\mathrm{i} E_{\mathrm{if}} \tau} \mathrm{e}^{\mathrm{i}\left(E_{v}^{\mathrm{i}}-E_{\mu}^{\mathrm{f}}\right) \tau}
$$

其中 $\tau \equiv t / \hbar$

所以, 在谐振子近似下, 将式(13)和(14)代入(5) (7)可以得到:

$$
\begin{aligned}
& k_{\mathrm{f} \leftarrow \mathrm{i}}^{(0)}=\frac{1}{\hbar^{2}} R_{\mathrm{fi}}^{\mathrm{isc}} \int_{-\infty}^{\infty} \mathrm{d} t \mathrm{e}^{\mathrm{i} E_{\mathrm{iif}} t / \hbar} Z_{\mathrm{i}}^{-1} \rho_{\mathrm{fi}}^{(0)}(t) \\
& k_{\mathrm{f} \leftarrow \mathrm{i}}^{(1)}=2 \operatorname{Re}\left[\frac{1}{\hbar^{2}} \int_{-\infty}^{\infty} \mathrm{d} t \mathrm{e}^{\mathrm{i} E_{\mathrm{ii}} t / \hbar} Z_{\mathrm{i}}^{-1} \sum_{k} R_{\mathrm{fi}, k}^{\mathrm{isc}} \rho_{\mathrm{fi}, k}^{(1)}(t)\right] \\
& k_{\mathrm{f} \leftarrow \mathrm{i}}^{(2)}=\frac{1}{\hbar^{2}} \int_{-\infty}^{\infty} \mathrm{d} t \mathrm{e}^{\mathrm{i} E_{\mathrm{if}} t / \hbar} Z_{\mathrm{i}}^{-1} \sum_{k, l} R_{\mathrm{fi}, k l}^{\mathrm{isc}} \rho_{\mathrm{fi}, k l}^{(2)}(t)
\end{aligned}
$$

其中

$$
\begin{aligned}
& \rho_{\mathrm{fi}}^{(0)}(t) \equiv \operatorname{Tr}\left[\mathrm{e}^{-\mathrm{i} \tau_{\mathrm{f}} \hat{H}^{\mathrm{f}}} \mathrm{e}^{-\mathrm{i} \tau_{\mathrm{i}} \hat{H}^{\mathrm{i}}}\right] \\
& \rho_{\mathrm{fi}, k}^{(1)}(t) \equiv \operatorname{Tr}\left[\hat{P}_{\mathrm{fk}} \mathrm{e}^{-\mathrm{i} \tau_{\mathrm{f}} \hat{H}^{\mathrm{f}}} \mathrm{e}^{-\mathrm{i} \tau_{\mathrm{i}} \hat{H}^{\mathrm{i}}}\right] \\
& \rho_{\mathrm{fi}, k l}^{(2)}(t) \equiv \operatorname{Tr}\left[\hat{P}_{\mathrm{fk}} \mathrm{e}^{-\mathrm{i} \tau_{\mathrm{f}} \hat{H}^{\mathrm{f}}} \hat{P}_{\mathrm{fl}} \mathrm{e}^{-\mathrm{i} \tau_{\mathrm{i}} \hat{H}^{\mathrm{i}}}\right]
\end{aligned}
$$

和 $\tau_{\mathrm{f}} \equiv \tau, \tau_{\mathrm{i}} \equiv-\tau-\mathrm{i} \beta$.

考虑到初、末两电子态势能面的不同与联系:

$$
Q_{\mathrm{i} l}=\sum_{k} S_{l k} Q_{\mathrm{f} k}+D_{l}
$$

$S_{l k}$ 表示初态第 $l$ 个模式与末态第 $k$ 个模式的混合程 度(Duschinsky 矩阵 $\mathbf{S}$ 的一个矩阵元素), $D_{l}$ 表示第 $l$ 个 模式的位移.

利用多维高斯积分及其导数, 可以求解关联函数得 到解析形式:

$$
\begin{gathered}
\rho_{\mathrm{fi}}^{(0)}(t)=\sqrt{\frac{\operatorname{det}\left[\mathbf{a}_{\mathrm{f}} \mathbf{a}_{\mathrm{i}}\right]}{\operatorname{det}[\mathbf{K}]}} \exp \left\{\frac{i}{\hbar}\left[-\frac{1}{2} \underline{F}^{\mathrm{T}} \boldsymbol{K}^{-1} \underline{F}+\underline{D}^{\mathrm{T}} \boldsymbol{E} \underline{D}\right]\right\} \\
\rho_{\mathrm{fi}, k}^{(1)}(t)=-\underline{H}_{k}^{\mathrm{T}} \mathbf{K}^{-1} \underline{F} \sqrt{\frac{\operatorname{det}\left[\mathbf{a}_{\mathrm{f}} \mathbf{a}_{\mathrm{i}}\right]}{\operatorname{det}[\mathbf{K}]}} \\
\exp \left\{\frac{i}{\hbar}\left[-\frac{1}{2} \underline{F}^{\mathrm{T}} \boldsymbol{K}^{-1} \underline{F}+\underline{D}^{\mathrm{T}} \boldsymbol{E} \underline{D}\right]\right\} \\
\rho_{\mathrm{fi}, k l}^{(2)}(t)=\rho_{\mathrm{fi}}^{(0)}(t)\left\{\begin{array}{l}
\mathrm{i} \hbar \operatorname{Tr}\left[\mathbf{G}_{k l} \mathbf{K}^{-1}\right]+ \\
\left(\mathbf{K}^{-1} \underline{F}\right)^{\mathrm{T}} \mathbf{G}_{k l}\left(\mathbf{K}^{-1} \underline{F}\right)-\underline{H}_{k l}^{\mathrm{T}} \mathbf{K}^{-1} \underline{F}
\end{array}\right\}
\end{gathered}
$$

其中 $\mathbf{a}_{\mathrm{i}}$ 和 $\mathbf{a}_{\mathrm{f}}$ 是 $N \times N$ 的对角矩阵; $\mathbf{A}, \mathbf{B}$ 和 $\boldsymbol{E}$ 是 $N \times N$ 的矩阵; $\underline{\boldsymbol{F}}, \underline{\boldsymbol{H}}_{k}$ 和 $\underline{\boldsymbol{H}}_{k l}$ 为 $2 N \times 1$ 列矩阵, $\boldsymbol{K}$ 和 $\boldsymbol{G}_{k l}$ 是
$2 N \times 2 N$ 的矩阵. 详细的推导和矩阵含义可以参看文 献 ${ }^{[12,18,19]}$.

\section{2 计算细节}

公式(15 17)中的所有物理量包括基态和激发态的 振动模、绝热和垂直跃迁的电子激发态能级均可以通过 现代量子化学的方法, 不需要任何经验参数. $\mathrm{BO}$ 微扰矩 阵元和自旋-轨道耦合系数也可以分别求得. 首先, 我 们分别采用 B3LYP 和 UB3LYP 方法对有机过渡金属配 合物 $f a c-\operatorname{Ir}\left(\mathrm{F}_{2} \text { ppy }\right)_{3}$ 的基态 $\mathrm{S}_{0}$ 和第一激发态三线态 $\mathrm{T}_{1}$ 的 几何构型进行优化. 之后, 进行两电子态的优化构型下 的频率分析. 结果无虚频，证明得到的均为分子的平衡 构型. 以上计算中, $\mathrm{C}, \mathrm{H}, \mathrm{N}$ 和 $\mathrm{F}$ 原子使用 6-31 ${ }^{* *}$ 基组, Ir 原子使用 SDD 基组, 计算由 Gaussian 09 程序包 ${ }^{[20]}$ 完成.

基于优化的基态平衡构型，我们在Dalton程序包 ${ }^{[21]}$ 里采用密度泛函理论的线性响应和二次响应理论分别 计算了两电子态之间的自旋轨道耦合常数和跃迁偶极 矩 ${ }^{[22,23]}$. 其中, 我们在自旋轨道耦合常数计算中使用了 有效单电子近似 ${ }^{[24,25]}$, 此近似已经得到广泛的应 用 ${ }^{[26-29]}$; 所用的基组同上.

在一阶微扰的框架下, 非绝热耦合矩阵元可以表示 为:

$$
\left\langle\Phi_{\mathrm{f}} \mid \frac{\partial \Phi_{\mathrm{i}}}{\partial Q_{\mathrm{f} k}}\right\rangle \approx \frac{\left\langle\Phi_{\mathrm{f}}^{0}\left|\frac{\partial V}{\partial Q_{\mathrm{f} k}}\right| \Phi_{\mathrm{i}}^{0}\right\rangle}{E\left(\Phi_{\mathrm{i}}^{0}\right)-E\left(\Phi_{\mathrm{f}}^{0}\right)}
$$

通过将正则模式变换到笛卡尔坐标，耦合元能转换 成在对所有原子核处点电荷的电场求和, 从而可以在量 子化学水平上计算 ${ }^{[18]}$.

根据两电子态的电子结构信息, 根据 Eckart 转动条 件确定两电子态平衡位形关系, 采用笛卡尔坐标系计算 出体系的 Duschinsky 转动矩阵和位移矢量 ${ }^{[18]}$. 最后, 结 合分子的跃迁性质, 使用本组自主发展的光谱和速率理 论程序，计算出有机过渡金属配合物 $f a c-\operatorname{Ir}\left(\mathrm{F}_{2} \mathrm{ppy}\right)_{3}$ 在不 同温度下的磷光光谱、辐射衰减速率和系间窝越速率、 磷光效率及激发态寿命.

\section{3 结果与讨论}

\section{1 基态和激发态构型参数}

图 1 给出了化合物 $f a c-\operatorname{Ir}\left(\mathrm{F}_{2} \mathrm{ppy}\right)_{3}$ 的分子结构及原子 编号. 表 1 列出了 $\mathrm{S}_{0}$ 和 $\mathrm{T}_{1}$ 态重要的平衡构型参数. 由数 据可以看出: (1)基态平衡构型接近于 $C_{3}$ 对称点群; (2)电 子激发到三线态, 配体 L1 靠近了中心铱原子, 表现为 $\operatorname{Ir}(1)-\mathrm{C}(2)$ 和 $\operatorname{Ir}(1)-\mathrm{N}(45)$ 的键长明显减少; 而其余两个 配体 L2 和 L3 远离了中心铱原子, 表现为 $\operatorname{Ir}(1)-\mathrm{C}(10)$, $\operatorname{Ir}(1)-\mathrm{N}(29)$ 和 $\operatorname{Ir}(1)-\mathrm{N}(37)$ 键长的增加, 这样导致了化 合物对称性的破缺; (3)基态和激发态结构之间最大改变 量主要发生在配体 $\mathrm{L} 1$ 上，例如 $\mathrm{C}(5)-\mathrm{C}(46)$ 键长从 1.466 
$\AA$ 缩短到 $1.404 \AA, \mathrm{C}(46)-\mathrm{N}(45)$ 从 $1.367 \AA$ 增加到 1.435 $\AA$ 及 $\mathrm{C}(5)-\mathrm{C}(2)$ 键长从 1.434 增加到 $1.487 \AA$. 而其它两 个配体中的结构参数变化很小. 这预示着铱原子和配体 $\mathrm{L} 1$ 主要参与了 $\mathrm{T}_{1}$ 到 $\mathrm{S}_{0}$ 的光物理过程.

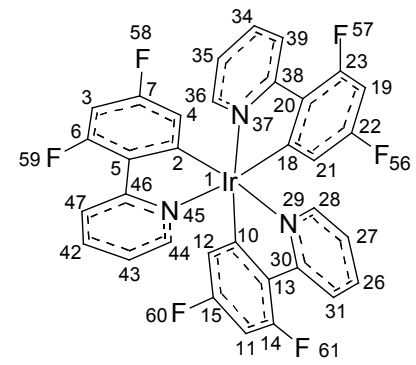

图 $1 \mathrm{fac}-\mathrm{Ir}\left(\mathrm{F}_{2} \mathrm{ppy}\right)_{3}$ 的分子结构和原子编号

Figure 1 The molecular structure and atom-numbering scheme of fac- $\operatorname{Ir}\left(\mathrm{F}_{2} \mathrm{ppy}\right)_{3}$

\section{2 前线分子轨道}

查看分析 $f a c-\operatorname{Ir}\left(\mathrm{F}_{2} \text { ppy }\right)_{3}$ 基态 $\mathrm{S}_{0}$ 的最高占据轨道 (HOMO) 和激发态 $\mathrm{T}_{1}$ 的 HOMO 的原子轨道组成可知: 基态的 $\mathrm{HOMO}$ 轨道一半来自于金属的 $\mathrm{d}$ 轨道 $(50.00 \%)$, 一半来自于三个配体的 $\pi$ 轨道; 激发态 $\mathrm{T}_{1}$ 的 $\mathrm{HOMO}$ 主 要是配体 $\mathrm{L} 1$ 的 $\pi$ 轨道的贡献 $(94.82 \%$, 其中氟化苯环占 $37.09 \%$, 吡啶环占 $57.73 \%$ ). 因此, 可以得出 $\mathrm{T}_{1}$ 与 $\mathrm{S}_{0}$ 态 之间主要发生了从金属到配体的跃迁 MLCT, 混合一部 分配体内 $\pi \rightarrow \pi^{*}$ 跃迁. 这同时表明，与只有 $\pi \rightarrow \pi^{*}$ 跃迁的
纯有机分子或者其它金属有机配合物，这类化合物将具 有强的自旋轨道耦合, 有利于 $\mathrm{T}_{1}$ 到 $\mathrm{S}_{0}$ 的跃迁. 另外, 由 于氟原子的强吸电子作用, $f a c-\operatorname{Ir}\left(\mathrm{F}_{2} \text { ppy }\right)_{3}$ 的 $\mathrm{T}_{1}$ 与 $\mathrm{S}_{0}$ 之间 的能隙 $(2.977 \mathrm{eV})$ 比无氟原子取代的 $f a c-\operatorname{Ir}(\mathrm{ppy})_{3}$ 的两态 间能隙 $(2.777 \mathrm{eV})$ 增加了 $0.200 \mathrm{eV}\left(1613.20 \mathrm{~cm}^{-1}\right)$, 这致 使光谱蓝移.

\section{3 光物理性质}

\subsection{1 发射光谱}

光谱通常是衡量一种新方法的基本手段. 我们首先 将理论计算与现有的实验光谱进行了比较, 如图 3a 所 示. 这里, 为了将计算最大峰位 $(433.49 \mathrm{~nm})$ 和实验测量 的最大发射峰位 $(450.75 \mathrm{~nm}$ )重叠, 我们把理论计算光谱 红移了 $17.26 \mathrm{~nm}$ (约 $0.1 \mathrm{eV}$ ). 由图 3a 可知, 两者光谱的 精细结构吻合得非常好, 说明我们选择的密度泛函方法 和自主发展的关联函数方法对这类有机金属配合物的 光物理性质描述是可靠和可行的. 我们进一步给出了化 合物的发射光谱与温度的依赖关系, 如图 3b 所示. 我们 可以看到: (1)低温下, 发射光谱具有更细的精细结构, 最大峰的来源主要是纯的电子态跃迁(0-0 跃迁); (2)随 着温度升高, 众多的振动态被激活, 抹平了精细结构, 展宽了光谱而减小了峰的强度，同时使得最大峰位发生 明显的红移; (3) $298 \mathrm{~K}$ 下的最大发射峰位是 $469.01 \mathrm{~nm}$, 与实验测量值 466 468 nm 非常接近(见表 2), 进一步 印证了预测方法的合理性.

表 1 化合物 $f a c-\mathrm{Ir}\left(\mathrm{F}_{2} \mathrm{ppy}\right)_{3}$ 在 $\mathrm{S}_{0}$ 和 $\mathrm{T}_{1}$ 态下的平衡构型参数：键长 $(\AA)$ 、键角 $\left({ }^{\circ}\right)$ 和二面角 $\left({ }^{\circ}\right)$

Table 1 Selected bond length $(\AA)$, bond angles and dihedral angles $\left(^{\circ}\right)$ in the $\mathrm{S}_{0}$ and $\mathrm{T}_{1}$ for $f a c-\operatorname{Ir}\left(\mathrm{F}_{2} \text { ppy }\right)_{3}$

\begin{tabular}{|c|c|c|c|c|c|c|c|c|}
\hline & Bond length & $\mathrm{S}_{0}$ & $\mathrm{~T}_{1}$ & $\Delta\left(\mathrm{T}_{1}-\mathrm{S}_{0}\right)$ & Bond angles and dihedral angles & $\mathrm{S}_{0}$ & $\mathrm{~T}_{1}$ & $\Delta\left(\mathrm{T}_{1}-\mathrm{S}_{0}\right)$ \\
\hline & $\operatorname{Ir}(1)-C(2)$ & 2.034 & 2.011 & -0.023 & $\operatorname{Ir}(1)-\mathrm{N}(45)-\mathrm{C}(46)$ & 114.7 & 113.7 & -1.0 \\
\hline & $\operatorname{Ir}(1)-N(45)$ & 2.177 & 2.133 & -0.044 & $C(2)-C(5)-C(6)$ & 118.5 & 116.6 & -1.9 \\
\hline & $C(5)-C(46)$ & 1.466 & 1.404 & -0.062 & $C(6)-C(3)-C(7)$ & 116.8 & 118.4 & 1.6 \\
\hline \multirow[t]{7}{*}{ L1 } & $\mathrm{C}(46)-\mathrm{N}(45)$ & 1.367 & 1.435 & 0.068 & $\mathrm{C}(44)-\mathrm{N}(45)-\mathrm{C}(46)$ & 120.1 & 118.8 & -1.3 \\
\hline & $\mathrm{C}(44)-\mathrm{N}(45)$ & 1.344 & 1.337 & -0.007 & $C(5)-C(46)-C(47)$ & 126.5 & 127.5 & 1.0 \\
\hline & $C(5)-C(2)$ & 1.434 & 1.487 & 0.053 & $\mathrm{C}(2)-\mathrm{C}(5)-\mathrm{C}(46)-\mathrm{N}(45)$ & -1.9 & 1.0 & 2.9 \\
\hline & $C(4)-C(2)$ & 1.405 & 1.397 & -0.008 & $\mathrm{C}(44)-\mathrm{N}(45)-\mathrm{C}(46)-\mathrm{C}(5)$ & 179.2 & 175.6 & -3.6 \\
\hline & $\operatorname{Ir}(1)-\mathbf{C}(10)$ & 2.035 & 2.049 & 0.014 & $\operatorname{Ir}(1)-\mathrm{N}(29)-\mathrm{C}(30)$ & 114.7 & 114.5 & -0.2 \\
\hline & $\operatorname{Ir}(1)-N(29)$ & 2.179 & 2.193 & 0.014 & $\mathrm{C}(10)-\mathrm{C}(13)-\mathrm{C}(14)$ & 118.6 & 118.4 & -0.2 \\
\hline & $\mathrm{C}(13)-\mathrm{C}(30)$ & 1.467 & 1.468 & 0.001 & $C(14)-C(11)-C(15)$ & 116.8 & 116.9 & 0.1 \\
\hline \multirow[t]{7}{*}{ L2 } & $\mathrm{C}(30)-\mathrm{N}(29)$ & 1.367 & 1.366 & -0.001 & $\mathrm{C}(28)-\mathrm{N}(29)-\mathrm{C}(30)$ & 120.1 & 120.5 & 0.4 \\
\hline & $\mathrm{C}(28)-\mathrm{N}(29)$ & 1.344 & 1.344 & 0 & $\mathrm{C}(13)-\mathrm{C}(30)-\mathrm{C}(31)$ & 126.5 & 126.4 & -0.1 \\
\hline & $\mathrm{C}(13)-\mathrm{C}(10)$ & 1.434 & 1.433 & -0.001 & $\mathrm{C}(10)-\mathrm{C}(13)-\mathrm{C}(30)-\mathrm{N}(29)$ & -1.8 & -1.9 & -0.1 \\
\hline & $\mathrm{C}(12)-\mathrm{C}(10)$ & 1.405 & 1.403 & -0.002 & $\mathrm{C}(28)-\mathrm{N}(29)-\mathrm{C}(30)-\mathrm{C}(13)$ & 179.2 & 179.6 & 0.4 \\
\hline & $\operatorname{Ir}(1)-C(18)$ & 2.036 & 2.031 & -0.005 & $\operatorname{Ir}(1)-\mathrm{N}(37)-\mathrm{C}(38)$ & 114.8 & 114.5 & -0.3 \\
\hline & $\operatorname{Ir}(1)-N(37)$ & 2.179 & 2.190 & 0.011 & $\mathrm{C}(18)-\mathrm{C}(20)-\mathrm{C}(23)$ & 118.5 & 118.3 & -0.2 \\
\hline & $\mathrm{C}(20)-\mathrm{C}(38)$ & 1.466 & 1.466 & 0 & C(23)-C(19)-C(22) & 116.8 & 116.9 & 0.1 \\
\hline \multirow[t]{4}{*}{ L3 } & $\mathrm{C}(38)-\mathrm{N}(37)$ & 1.367 & 1.365 & -0.002 & $\mathrm{C}(36)-\mathrm{N}(37)-\mathrm{C}(38)$ & 120.1 & 120.3 & 0.2 \\
\hline & $\mathrm{C}(36)-\mathrm{N}(37)$ & 1.344 & 1.343 & -0.001 & C(20)-C(38)-C(39) & 126.5 & 126.5 & 0 \\
\hline & $C(20)-C(18)$ & 1.434 & 1.433 & -0.001 & $\mathrm{C}(18)-\mathrm{C}(20)-\mathrm{C}(38)-\mathrm{N}(37)$ & -1.6 & -1.7 & -0.1 \\
\hline & $C(21)-C(18)$ & 1.405 & 1.404 & -0.001 & $\mathrm{C}(36)-\mathrm{N}(37)-\mathrm{C}(38)-\mathrm{C}(20)$ & 179.0 & 178.6 & -0.4 \\
\hline
\end{tabular}




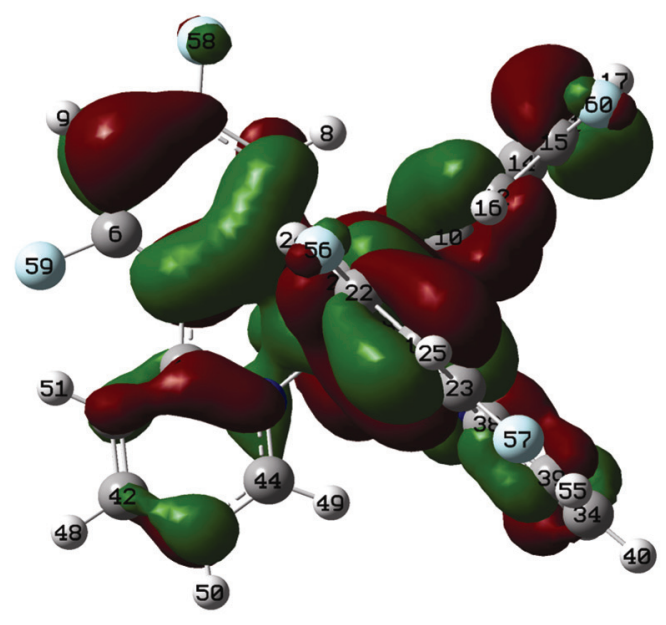

$\mathrm{S}_{0}: \mathrm{HOMO}$

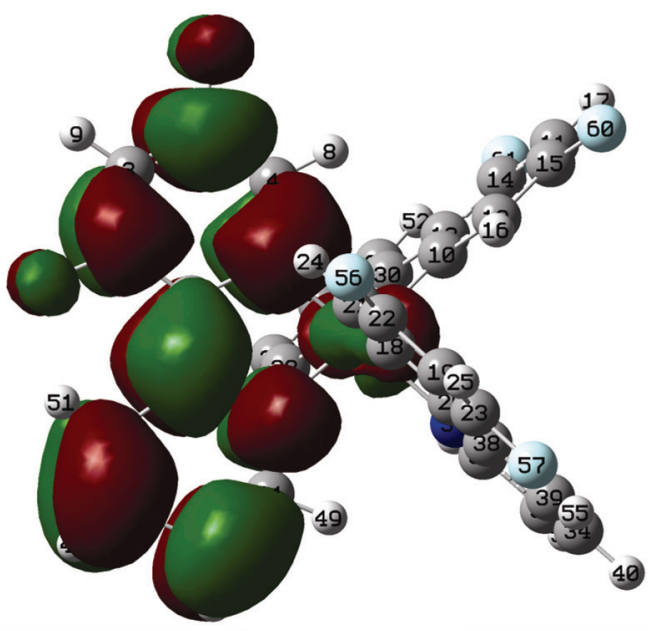

$\mathrm{T}_{1}: \mathrm{HOMO}$

图 2 计算所得到的 $f a c-\operatorname{Ir}\left(\mathrm{F}_{2} \mathrm{ppy}\right)_{3}$ 的基态 $\mathrm{S}_{0}$ 和激发态 $\mathrm{T}_{1}$ 的 $\mathrm{HOMO}$

Figure 2 Calculated HOMOs in the electronic states $\mathrm{S}_{0}$ and $\mathrm{T}_{1}$ for $f a c-\operatorname{Ir}\left(\mathrm{F}_{2} \mathrm{ppy}\right)_{3}$
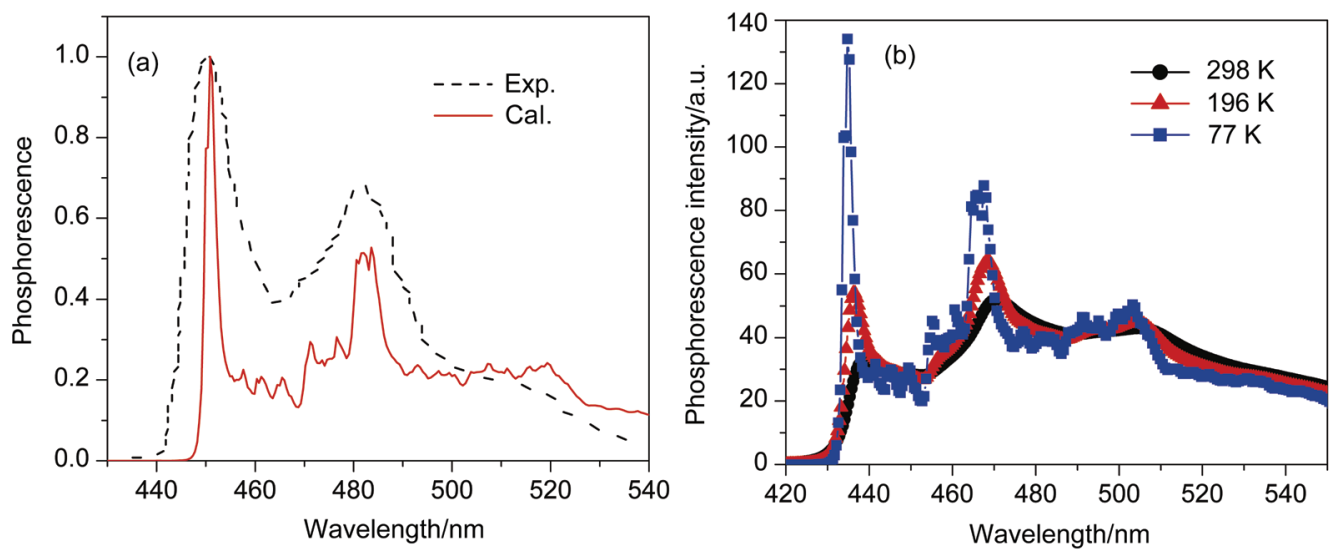

图 $3 \mathrm{fac}-\mathrm{Ir}\left(\mathrm{F}_{2} \mathrm{ppy}\right)_{3}$ 的磷光光谱: (a) $77 \mathrm{~K}$ 下, 计算得到的归一化磷光光谱与在 2-MeTHF 溶液中的实验光谱的比较; (b) 不同温度下的磷光光谱 Figure 3 Phosphorescence spectra for $f a c-\operatorname{Ir}\left(\mathrm{F}_{2} \mathrm{ppy}\right)_{3}$ : (a) Comparison between the calculated normalized spectrum and the experiment measured in 2-MeTHF at $77 \mathrm{~K}$; (b) Calculated phosphorescence spectra at different temperatures

\subsection{2 辐射和无辐射跃迁速率}

$\mathrm{T}_{1}$ 具有三个不同能量的子电子态 $\mathrm{T}_{1, m=-1,0,1}$. 在较低 温度下, 电子跃迁的起始态是能量最低的 $\mathrm{T}_{1, m=-1}$; 随着 温度的升高, 能量稍高的 $\mathrm{T}_{1, m}=0$ 的发射峰出现; 随后, 能 量最高的 $\mathrm{T}_{1, m=1}$ 的发射峰出现. 这些可以在低于 $10 \mathrm{~K}$ 的 超精细光谱中观测到 ${ }^{[30]}$. 温度再升高, 三个子电子态会 按照一定方式分布, 像波尔兹曼分布, 不可区别. 当温 度升高到一定值(对 $f a c-\operatorname{Ir}\left(\mathrm{F}_{2} \mathrm{ppy}\right)_{3}$ 来说是 $200 \mathrm{~K}$ ), 三个子 电子态几乎成平均分布, 不再依赖于三个子电子态的零 场䢃裂值(ZFS, zero field splitting $)^{[7]}$. 所以, 在以下跃迁 速率计算中, 我们简单地对三个子电子态作平均. 辐射 跃迁速率是简单的对发射光谱在整个范围内的积分. 无 辐射跃迁过程中, MLCT 的发生使得 $\mathrm{T}_{1}$ 与 $\mathrm{S}_{0}$ 之间的自旋 轨道耦合值 $\left(58.56 \mathrm{~cm}^{-1}\right)$ 远大于纯有机分子的自旋轨道 耦合值 $\left(<0.1 \mathrm{~cm}^{-1}\right)$. 这就使得无辐射跃迁公式中的一 阶微扰项远大于二阶微扰项. 所以, 无辐射跃迁速率计 算中, 只考虑一阶微扰项. 计算出的 $f a c-\operatorname{Ir}\left(\mathrm{F}_{2} \mathrm{ppy}\right)_{3}$ 的光
物理性质见表 2 , 同时给出了实验测量值. 无论是磷光 光谱最大峰位还是辐射速率、无辐射衰减速率和磷光效 率均与实验值吻合得非常好. 这表明, 热关联函数方法 成功实现了完全基于第一性原理的对有机过渡金属配 合物的光物理性质的定量预测.

表 2 化合物 $f a c-\operatorname{Ir}\left(\mathrm{F}_{2} \mathrm{ppy}\right)_{3}$ 的光物理性质

Table 2 Photophysical properties of $f a c-\operatorname{Ir}\left(\mathrm{F}_{2} \text { ppy }\right)_{3}$ at $298 \mathrm{~K}$

\begin{tabular}{lllll}
\hline & $\lambda_{\max } / \mathrm{nm}$ & $\Phi_{p}$ & $k_{\mathrm{r}} / \mathrm{s}^{-1}$ & $k_{\mathrm{nr}} / \mathrm{s}^{-1}$ \\
\hline Cal. & $\mathbf{4 6 9 . 1 0}$ & $\mathbf{0 . 7 6}$ & $\mathbf{2 . 8 1} \times \mathbf{1 0}$ & $\mathbf{8 . 9} \times \mathbf{1 0}^{\mathbf{5}}$ \\
Exp. $^{a}$ & 466 & 0.98 & $5.8 \times 10^{5}$ & $1.2 \times 10^{4}$ \\
Exp. $^{b}$ & 469 & 0.77 & $4.7 \times 10^{5}$ & $1.4 \times 10^{5}$ \\
Exp. $^{c}$ & 468 & 0.43 & $2.7 \times 10^{5}$ & $3.6 \times 10^{5}$ \\
\hline
\end{tabular}

${ }^{a}$ in 2-MeTHF solution, ref. [7]; ${ }^{b}$ in 2-MeTHF glass, ref. [14]; ${ }^{c}$ in $\mathrm{CH}_{2} \mathrm{Cl}_{2}$, ref. [15].

我们进一步给出了辐射、无辐射衰减速率及激发态 寿命与温度的依赖关系, 如图 4 所示. 我们可以看到, 随着温度的升高, 辐射衰减速率呈现出稍微的减小趋 
势, 而无辐射衰减速率明显增加. 这导致了激发态寿命 随着温度升高是降低的, 与实验观测结果一致 ${ }^{[7]}$.
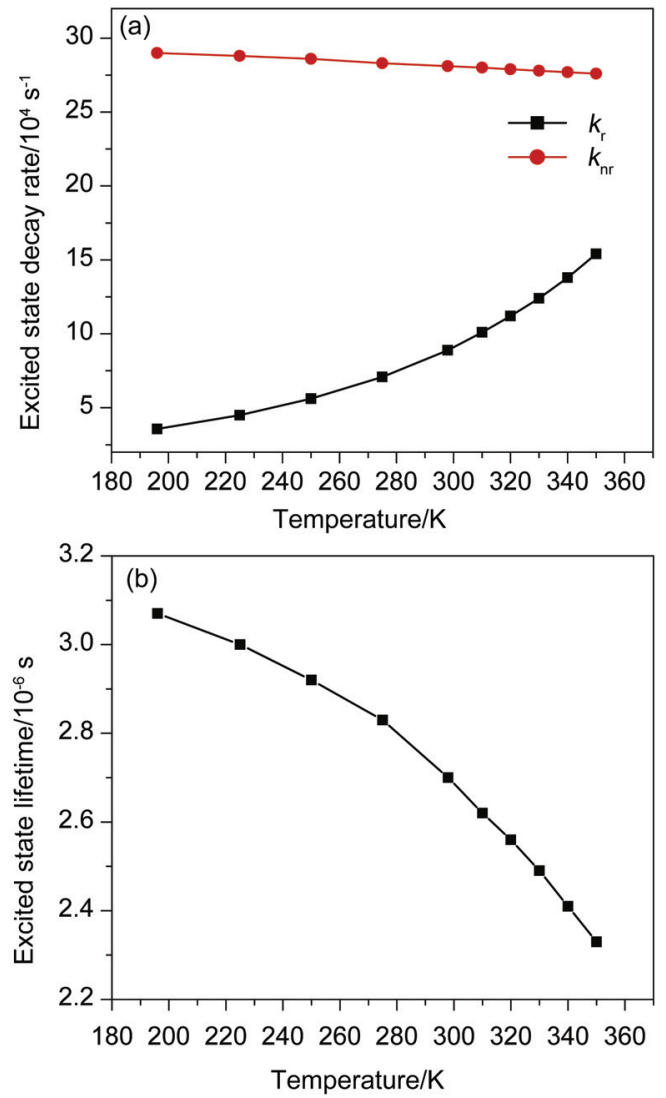

图 4 (a) 辐射跃迁速率 $k_{\mathrm{r}}$ 和无辐射跃迁速率 $k_{\mathrm{nr}}$ 与温度的依赖关系; (b)激发态寿命与温度的依赖关系

Figure 4 (a) Temperature dependence of the radiative and nonradiative transition rates; (b) Temperature dependence of the excited state lifetime

\subsection{3 无辐射衰减途径分析}

无辐射衰减过程就是把过多的激发电子态能量转 变为分子的热振动. 由此可知, 电子与原子核振动的耦 合大小决定着无辐射衰减的途径. 而重整能 $(\lambda)$ 是从一 个电子态到另一个电子态的跃迁过程中分子结构弛豫 导致的能量变化, 是直接反映两电子态之间的电声子耦 合程度的物理量, 其大小也就标示着激发态衰减过程中 的无辐射途径. 在简谐近似下, 分子的重整能可以看成 是各个正则模式的弛豫总和: $\lambda=\sum_{l} S_{l} \hbar \omega_{l}=\sum_{l} \frac{1}{2} \omega_{l}^{2} D_{l}^{2}, S_{l}$ 是第 $l$ 个模式的黄昆系数, $D_{l}$ 是两个电子态间第 $l$ 个模式的原点位移.

图 5 给出了分子的正则模式对重整能的贡献. 由图 可以看出, 具有最大重整能的振动模式出现在高频模 式, 模式 69,149,150 和 153, 其振动频率分别为 703.19, $1528.40,1578.70$ 和 $1614.31 \mathrm{~cm}^{-1}$. 形象起见, 我们在图 6 中画出这些模式的位移向量. 从图中可以看出, 这些 重要的模式都出现在配体 L1 上, 这正好对应于上述的 几何构型参数变化规律和 $\mathrm{T}_{1}$ 到 $\mathrm{S}_{0}$ 的跃迁规律. 模式 69
是氟化苯环和吡啶环的面内呼吸模式; 模式 149 是连接 氟化苯环和吡啶环的 $\mathrm{C}(5)-\mathrm{C}(46)$ 键的伸缩振动及吡啶 环内 $\mathrm{C}(43)-\mathrm{C}(44)$ 键的伸缩振动; 模式 150 主要是吡啶 环内 $\mathrm{C}(42)-\mathrm{C}(47)$ 键的伸缩振动; 模式 153 是氟化苯环 内的 $\mathrm{C}(3)-\mathrm{C}(6)$ 键的伸缩振动. 这里, 取代氟原子 $\mathrm{F}$ 没 有直接参与有效的分子振动, 只是通过 $\mathrm{F}-\mathrm{C}$ 键稍微增 加了 $\mathrm{C}(3)-\mathrm{C}(6)$ 键振动能量, 所以相对于无 $\mathrm{F}$ 取代的 $\operatorname{Ir}(\mathrm{ppy})_{3}$ 来说, $\mathrm{F}$ 取代不会给 $f a c-\operatorname{Ir}\left(\mathrm{F}_{2} \mathrm{ppy}\right)_{3}$ 的无辐射衰减 速率带来很大影响.

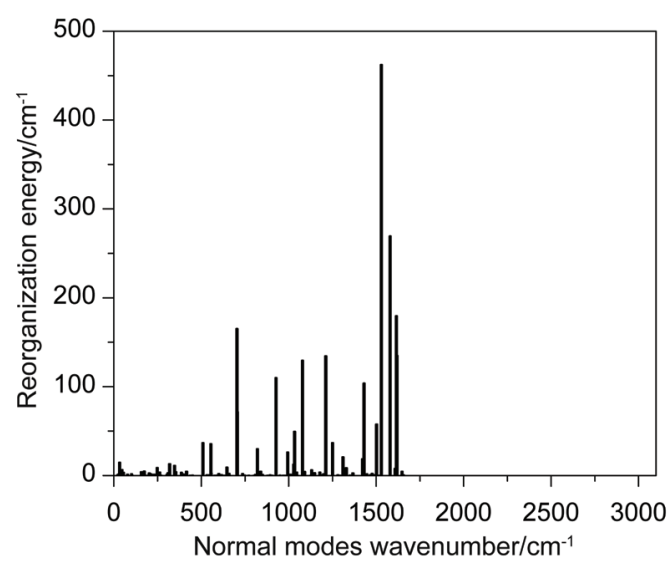

图 5 每个正则模式对重整能的贡献

Figure 5 Calculated reorganization energies versus the normal mode wavenumbers

由于正则模式反映的是分子整体的贡献，为了更好 地建立起结构性能关系，我们进一步把重整能在分子的 内坐标上进行分解. 图 7 给出前几个具有最大重整能的 内坐标, 同时给出相应的结构弛豫参数. 结合图 6 中正 则模式的位移向量, 我们可以看得出: 最大的重整能 (449.60 $\mathrm{cm}^{-1}$ ) 来源于 $\mathrm{C}(5)-\mathrm{C}(46)$ 键伸缩模式(1528.40 $\left.\mathrm{cm}^{-1}\right)$, 对应于从 $\mathrm{T}_{1}$ 到 $\mathrm{S}_{0}$ 很大的结构变化 $(-0.062 \AA)$. 类似地, 我们可以建立起其它坐标、振动模式及重整能 等之间的结构性能关系.

\section{4 结论}

结合密度泛函理论计算，运用我们最新自主发展的 振动关联函数计算公式, 详细计算研究了有机过渡金属 配合物 $f a c-\operatorname{Ir}\left(\mathrm{F}_{2} \text { ppy }\right)_{3}$ 的光物理性质，包括不同温度下的 磷光光谱、辐射跃迁速率、无辐射跃迁速率、磷光效率 及激发态寿命, 给出了与实验一致的结果. 系统分析了 影响化合物 $f a c-\operatorname{Ir}\left(\mathrm{F}_{2} \mathrm{ppy}\right)_{3}$ 光物理性质的因素: (1)取代基 氟原子调节了 $\mathrm{T}_{1}$ 与 $\mathrm{S}_{0}$ 之间的能隙, 相较于无取代的绿 光材料 $f a c-\operatorname{Ir}(\mathrm{ppy})_{3}$ ，发射波长发生蓝移，成为蓝光材料; (2)对重整能贡献很大的正则模式中没有氟原子的参与, 所以氟原子的引入没有增大无辐射衰减速率, 即不会很 大程度上降低其磷光效率; (3)磷光光谱与温度的依赖关 系：随着温度升高，众多的振动态被激活，抹平了低温 


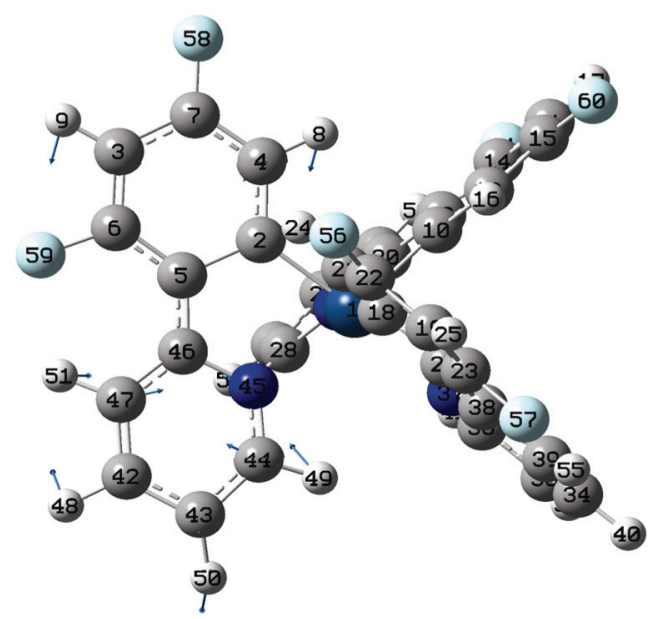

$69\left(703.19 \mathrm{~cm}^{-1}\right)$

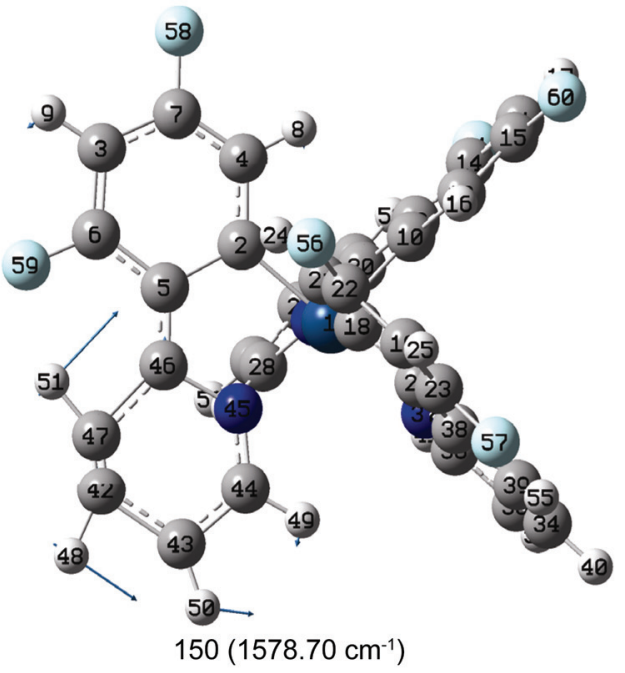

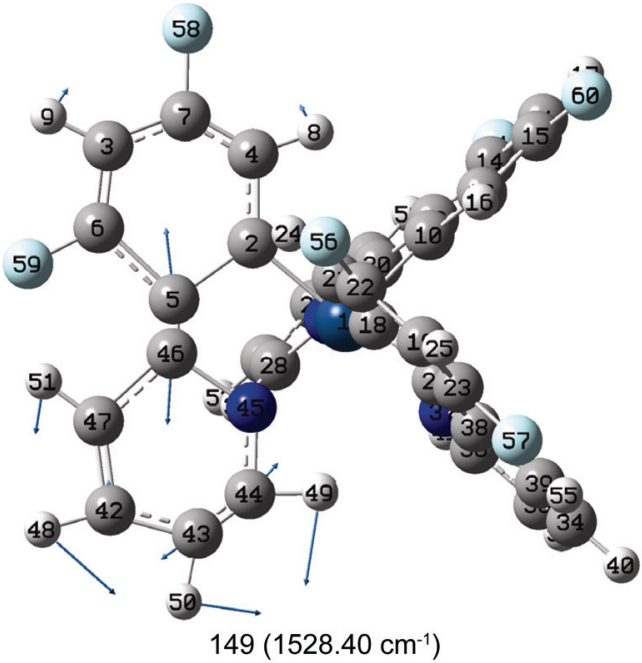

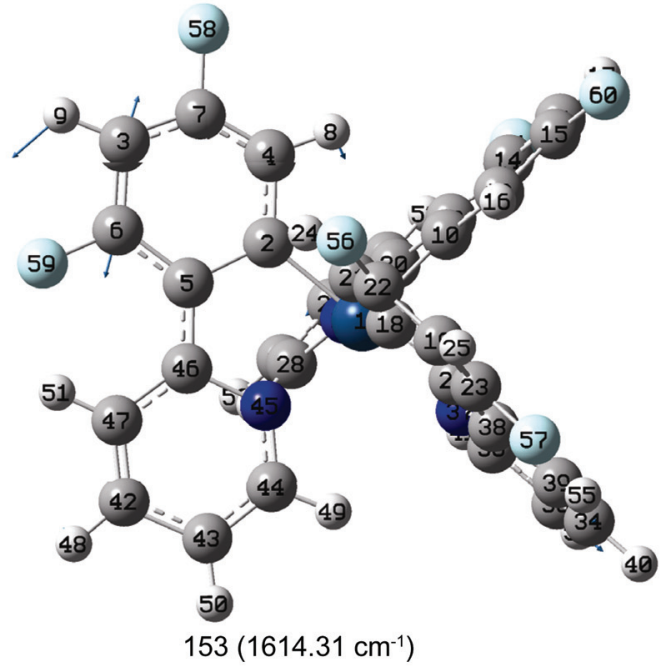

图 $6 f a c-\operatorname{Ir}\left(\mathrm{F}_{2} \mathrm{ppy}\right)_{3}$ 重要正则振动模式的位移向量示意图

Figure 6 Diagrammatic illustrations of the displacement vectors of the selected vibrational normal modes for $f a c-\operatorname{Ir}\left(\mathrm{F}_{2} \mathrm{ppy}\right)_{3}$

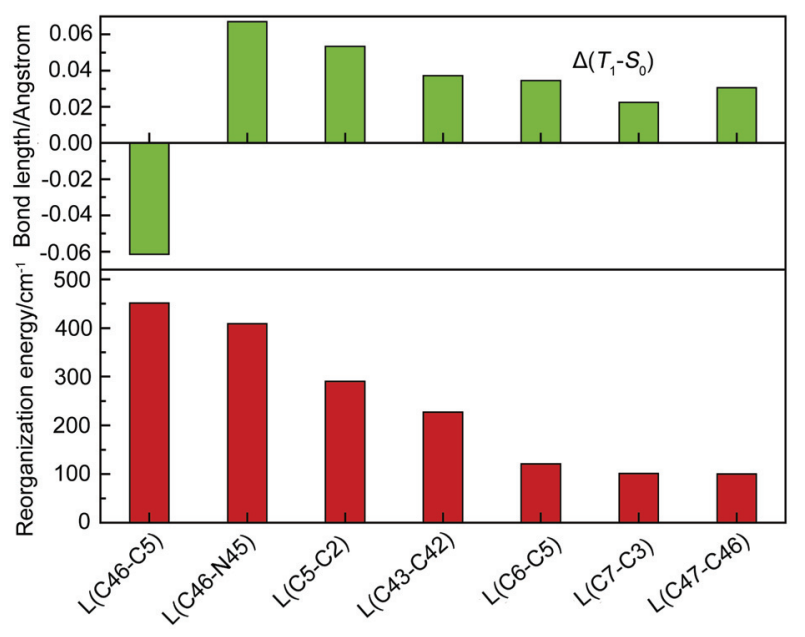

图 7 重整能在内坐标上的分解

Figure 7 Decomposition of the reorganization energies in the internal coordinates
下显现的精细结构, 展宽了光谱而减小了峰的强度, 同 时使得最大峰位发生明显的红移; (4) $f a c-\operatorname{Ir}\left(\mathrm{F}_{2} \mathrm{ppy}\right)_{3}$ 是高 效率的蓝色磷光材料; (5)激发态寿命随着温度的升高而 减小, 主要是无辐射衰减速率随着温度升高而增加的缘 故; (6)无辐射耗散激发态能量的主要途径是配体 L1 上 的碳碳键的伸缩振动. 例如, 连接氟化苯环和吡啶环的 $\mathrm{C}(5)-\mathrm{C}(46)$ 键、吡定环内 $\mathrm{C}(43)-\mathrm{C}(44)$ 键和 $\mathrm{C}(42)-$ $\mathrm{C}(47)$ 键及氟化苯环内的 $\mathrm{C}(3)-\mathrm{C}(6)$ 键. 以上信息均给分 子改造和设计进而合成更高效的磷光材料提供了理论 依据. 同时表明, 基于第一性原理, 无需任何经验参数, 运用我们的自主发展的光谱和速率理论, 可实现对有 机一过渡金属配合物的光物理性能的定量预测.

由于本工作所有的计算均在气相下进行，从而无法 解释表 2 中实验所给出的溶剂效应，这是将来要考虑的 一个发展方向. 


\section{致谢}

作者感谢牛英利博士在解析推导和程序编制方面 的大力帮助.

\section{References}

[1] Sun, Y.; Giebink, N. C.; Kanno, H.; Ma, B.; Thompson, M. E.; Forrest, S. R. Nature 2006, 440, 908.

[2] Lamansky, S.; Djurovich, P.; Murphy, D.; Abdel-Razzaq, F.; Lee, H.; Adachi, C.; Burrows, P. E.; Forrest, S. R.; Thompson, M. E. J. Am. Chem. Soc. 2001, 123(18), 4304

[3] Kwong, R. C.; Nugent, M. R.; Michalski, L.; Ngo, T.; Rajan, K.; Tung, Y.; Weaver, M. S.; Zhou, T. X.; Hack, M.; Thompson, M. E.; Forrest, S. R.; Brown, J. J. Appl. Phys. Lett. 2002, 81(1), 162.

[4] Tokito, S.; Iijima, T.; Tsuzuki, T.; Sato, F. Appl. Phys. Lett. 2003, 83(12), 2459.

[5] Tsuboyama, A.; Iwawaki, H.; Furugori, M.; Mukaide, T.; Kamatani, J.; Igawa, S.; Moriyama, T.; Miura, S.; Takiguchi, T.; Okada, S.; Hoshino, M.; Ueno, K. J. Am. Chem. Soc. 2003, 125(42), 12971.

[6] Baldo, M. A.; O'Brien, D. F.; You, Y.; Shoustikov, A.; Sibley, S.; Thompson, M. E.; Forrest, S. R. Nature 1998, 395(6698), 151.

[7] Sajoto, T.; Djurovich, P. I.; Tamayo, A. B.; Oxgaard, J.; Goddard, W. A.; Thompson, M. E. J. Am. Chem. Soc. 2009, 131(28), 9813.

[8] Endo, A.; Suzuki, K.; Yoshihara, T.; Tobita, S.; Yahiro, M.; Adachi, C. Chem. Phys. Lett. 2008, 460(1 3), 155.

[9] Wang, L. X.; Mei, Q. B.; Yan, F.; Tian, B.; Weng, J. N.; Zhang, B.; Huang, W. Acta Phys.-Chim. Sin. 2012, 28(7), 1556. (王玲霞，梅群 波, 颜芳, 田波, 翁洁娜, 张涁, 黄维, 物理化学学报, 2012, 28(7), 1556.)

[10] Tao, R.; Qiao, J.; Duan, L.; Qiu, Y. Prog. Chem. 2010, 22(12), 2255. (陶然, 乔娟, 段炼, 邱勇, 化学进展, 2010, 22(12), 2255.)

[11] Jansson, E.; Minaev, B.; Schrader, S.; Ågren, H. Chem. Phys. 2007, $333(2 \sim 3), 157$.

[12] Peng, Q.; Niu, Y.; Shi, Q.; Gao, X.; Shuai, Z. J. Chem. Theory Comput. 2013, 9(2), 1132.

[13] Jiang, Y. Q.; Peng, Q.; Gao, X.; Shuai, Z. G.; Niu, Y. L.; Lin, S. H. J. Mater. Chem. 2012, 22, 4491.

[14] Tamayo, A. B.; Alleyne, B. D.; Djurovich, P. I.; Lamansky, S.; Tsyba, I.; Ho, N. N.; Bau, R.; Thompson, M. E. J. Am. Chem. Soc. 2003, 125(24), 7377.

[15] Dedeian, K.; Shi, J.; Shepherd, N.; Forsythe, E.; Morton, D. C. Inorg. Chem. 2005, 44(13), 4445.
[16] Park, N. G.; Choi, G. C.; Lee, Y. H.; Kim, Y. S. Curr. Appl. Phys. 2006, 6(4), 620.

[17] Lin, S. H.; Chang, C. H.; Liang, K. K.; Chang, R.; Shiu, Y. J.; Zhang, J. M.; Yang, T. S.; Hayashi, M.; Hsu, F. C. Adv. Chem. Phys. 2002, 1.

[18] Niu, Y.; Peng, Q.; Deng, C.; Gao, X.; Shuai, Z. J. Phys. Chem. A 2010, 114(30), 7817

[19] Ianconescu, R.; Pollak, E. J. Phys. Chem. A 2004, 108(39), 7778.

[20] Frisch, M. J.; Trucks, G. W.; Schlegel, H. B.; Scuseria, G. E.; Robb, M. A.; Cheeseman, J. R.; Scalmani, G.; Barone, V.; Mennucci, B.; Petersson, G. A.; Nakatsuji, H.; Caricato, M.; Li, X.; Hratchian, H. P.; Izmaylov, A. F.; Bloino, J.; Zheng, G.; Sonnenberg, J. L.; Hada, M.; Ehara, M.; Toyota, K.; Fukuda, R.; Hasegawa, J.; Ishida, M.; Nakajima, T.; Honda, Y.; Kitao, O.; Nakai, H.; Vreven, T.; Montgomery, Jr., J. A.; Peralta, J. E.; Ogliaro, F.; Bearpark, M.; Heyd, J. J.; Brothers, E.; Kudin, K. N.; Staroverov, V. N.; Kobayashi, R.; Normand, J.; Raghavachari, K.; Rendell, A.; Burant, J. C.; Iyengar, S. S.; Tomasi, J.; Cossi, M.; Rega, N.; Millam, J. M.; Klene, M.; Knox, J. E.; Cross, J. B.; Bakken, V.; Adamo, C.; Jaramillo, J.; Gomperts, R.; Stratmann, R. E.; Yazyev, O.; Austin, A J.; Cammi, R.; Pomelli, C.; Ochterski, J. W.; Martin, R. L.; Morokuma, K.; Zakrzewski, V. G.; Voth, G. A.; Salvador, P.; Dannenberg, J. J.; Dapprich, S.; Daniels, A. D.; Farkas, Ö.; Foresman, J. B.; Ortiz, J. V.; Cioslowski, J.; Fox, D. J. Gaussian09, Revision B.01; Gaussian Inc., Wallingford, CT, 2010.

[21] Dalton, a molecular electronic structure program, Release Dalton2011 (2011), see http://daltonprogram.org.

[22] Minaev, B.; Ågren, H. Chem. Phys. 2005, 315(3), 215.

[23] Vahtras, O.; Ågren, H.; Jorgensen, P.; Jensen, H. J. A.; Helgaker, T.; Olsen, J. J. Chem. Phys. 1992, 97(12), 9178.

[24] Koseki, S.; Fedorov, D. G.; Schmidt, M. W.; Gordon, M. S. J. Phys. Chem. A 2001, 105(35), 8262.

[25] Koseki, S.; Schmidt, M. W.; Gordon, M. S. J. Phys. Chem. A 1998 , 102(50), 10430

[26] Li, X.; Minaev, B.; Ågren, H.; Tian, H. J. Phys. Chem. C 2011, $115(42), 20724$.

[27] Minaev, B.; Ågren, H.; Angelis, F. D. Chem. Phys. 2009, 358(3), 245.

[28] Minaev, B.; Minaeva, V.; Ågren, H. J. Phys. Chem. A 2009, 113(4), 726.

[29] Li, X.; Minaev, B.; Ågren, H.; Tian, H. Eur. J. Inorg. Chem. 2011, 2011(16), 2517.

[30] Yersin, H.; Finkenzeller, W. J. In Highly Efficient OLEDs with Phosphorescent Materials, Ed.: Yersin, H., Wiley-VCH Verlag GmbH \& Co. KgaA, Weiheim, Germany, 2008, pp. 1 98. 